Pecvnia, 6 (2008), pp. 53-73

\title{
El transporte de mercancías por carretera en Castilla y León
}

Recibido: Mayo 2008

Aceptado: Junio 2008

\section{Cristina Álvarez Folgueras \\ cristina.afolgueras@unileon.es \\ Ana Pardo Fanjul \\ ana.pardo@unileon.es \\ Universidad de León}

Fac. de Ciencias Económicas y Empresariales Dpto. de Economía y Estadística Campus de Vegazana, $s / n$ 24071 León (España)
El transporte es una parte fundamental en la actividad de cualquier sociedad. Se ha convertido en imprescindible en la actividad tanto profesional como lúdica de los individuos y es un elemento crítico en las relaciones económicas de los territorios. Las políticas de inversión en infraestructuras, junto con las mejoras tecnológicas en los vehículos, han sido algunas de las principales razones que han convertido a la carretera en el modo más utilizado tanto para el transporte de viajeros como para el de mercancías. En este trabajo se pretende analizar la situación actual del transporte de mercancías por carretera en la Comunidad Autónoma de Castilla y León, haciendo un repaso de diferentes magnitudes
Transportation is a fundamental part in the activity of any society. It has become essential for the professional and leisure activities of the individuals and it is a critical element in the economic relationships of the territories. The investment policies in infrastructures, as well as the technological improvements of the vehicles, are some of the main reasons that have turned the road into the most used mean of transport for people as well as for freight. In this work, we intend to analyze the current situation of road freight in the Comunidad Autónoma of Castilla y León, making a revision of different magnitudes directly related to the sector, as its relative weigh in the total activity of the region, 
directamente relacionadas con el sector, como su peso relativo en la actividad total de la región, la dimensión del parque de vehículos, la estructura empresarial de las empresas dedicadas a la actividad, la configuración de los flujos o el análisis del tipo de mercancía transportada.

Palabras clave: transporte por carretera, transporte de mercancías, economía regional, Castilla y León. the number of vehicles, the entrepreneurial structure of the companies devoted to the activity, the configuration of the flows or the analysis of the type of merchandise transported.

Key words: road freight, regional economy, Castilla y León.

\section{EL TRANSPORTE EN NUESTRA SOCIEDAD}

El transporte representa una actividad esencial en nuestra sociedad, tanto desde el punto de vista de la oferta, al ser uno de los principales componentes del sector servicios como desde el punto de vista de la demanda, al ser cada vez más necesario en las actividades laborales o de ocio de la ciudadanía. Además, el transporte, y en concreto las infraestructuras a él asociadas, han constituido elementos esenciales de la política económica de todos los países y han contribuido, junto con otras actuaciones, al desarrollo económico y social de las comunidades, a la vertebración del territorio, a la integración y cohesión del espacio, y a la mejora de las condiciones de accesibilidad. Pero también el transporte es responsable de gran cantidad de impactos sociales y ambientales negativos, por ejemplo la contaminación atmosférica, el ruido, los accidentes, la pérdida de biodiversidad por la fragmentación de los hábitat, la congestión o la dependencia energética.

En términos muy generales, el valor añadido por el sector del transporte oscila de forma más o menos cíclica entre el $3 \%$ y el $5 \%$ del PNB y absorbe entre el $5 \%$ y el $8 \%$ del total del empleo asalariado de un país desarrollado. La inversión pública a él asociada representa entre el $2 \%$ y el $2,5 \%$ del PNB y puede aumentar hasta el 3,5\% en aquellos países que están implicados en procesos de creación y modernización de las infraestructuras asociadas al sector. La mejora de estas infraestructuras y la apertura de las fronteras han contribuido al crecimiento del transporte; crecimiento que también se relaciona con la mejora de su relación calidad/precio, la eliminación de barreras políticas y el crecimiento económico.

En el ámbito de la UE-15, el volumen de tráfico de mercancías está creciendo a una tasa incluso superior a la del crecimiento económico 
por los cambios en los modelos de producción y distribución. Y el informe de la Comisión Europea EU15 Energy and transport Outlook to 2030 prevé que la demanda de transporte de mercancías continúe creciendo a una tasa del 2,1\% anual en los próximos veinte años.

La carretera es el modo que experimenta un mayor crecimiento, pues se han pasado de transportar así el 52,1\% del total de mercancías en 1970 (488 billones de toneladas-km) a transportar el 75,5\% en 2001 (1.396 billones de tn-km), como se puede observar en el Gráfico 1. En contrapartida, ha disminuido la importancia del ferrocarril, que apenas mueve el $8 \%$ de la mercancía transportada. No obstante, entre los estados miembros se observan algunas divergencias en los tipos de transporte utilizados, que se explican por la diversidad geográfica, por diferencias en las mercancías transportadas y por las distintas políticas económicas puestas en práctica por cada uno de los gobiernos.

Gráfico 1: Distribución por modos del transporte de mercancías en Europa. 2001. Porcentajes

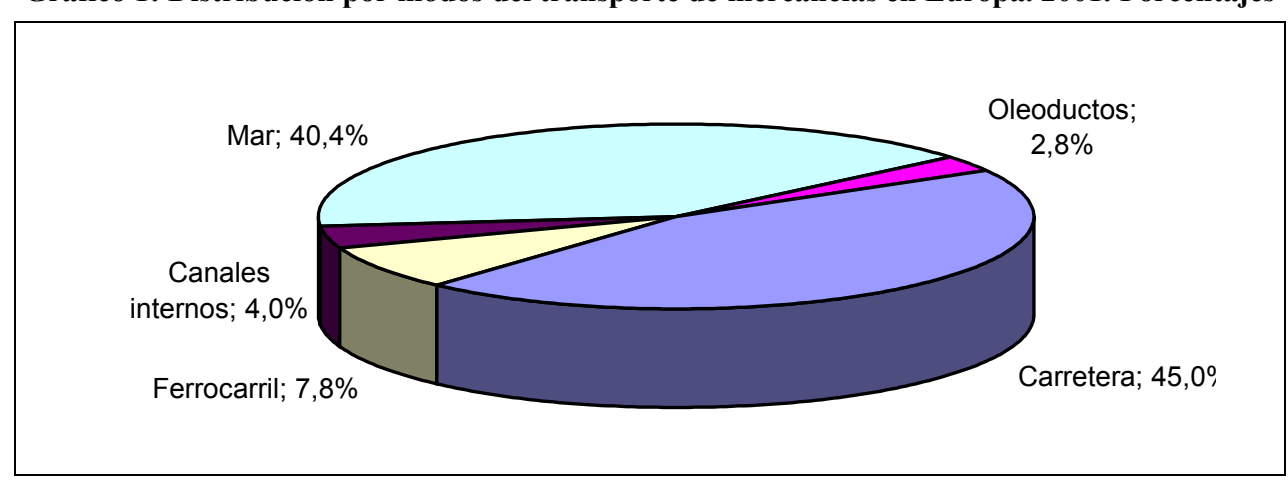

Fuente: European Foundation For the Improvement of Living and Working Conditions (2004).

Además de los citados con respecto al crecimiento general del sector, otros factores como el incremento de los ingresos (que se traduce en un aumento de la capacidad adquisitiva y de la propiedad de vehículos), las inversiones en infraestructuras (que han priorizado las carreteras), las mejoras tecnológicas (que generan menores precios y mayores velocidades de los vehículos) y el empeoramiento de la calidad del transporte público por ferrocarril, también explican el incremento del peso relativo de la carretera.

La política europea en esta materia aparece recogida en el Libro Blanco sobre la Política Común de Transportes. La Política Europea 
de Transportes de cara al 2010: la hora de la verdad (Comisión de las Comunidades Europeas 2001). La Unión Europea, preocupada por el cumplimiento del Protocolo de Kioto y por el mantenimiento o incremento de la competitividad del sector, plantea en este Libro Blanco unas 60 medidas que deberían ponerse en práctica a nivel comunitario de aquí a 2010. Su objetivo principal es la desconexión progresiva del crecimiento del transporte (y específicamente del transporte por carretera) del crecimiento económico, mediante un reequilibrio de los modos (revitalizando los alternativos a la carretera), la supresión de los puntos de estrangulamiento y el diseño de una política sectorial adecuada con incidencia en su seguridad y calidad.

siguientes:

Las principales medidas recogidas en el documento son las

- Revitalizar el ferrocarril

- Reforzar la calidad del transporte por carretera

- Fomentar el transporte marítimo y fluvial (autopistas del mar)

- Materializar la intermodalidad

- Reconciliar el crecimiento del transporte aéreo con el medio ambiente

- Realizar la red transeuropea de transportes

- Reforzar la seguridad vial

- Decidir una política de tarificación eficaz de los transportes

- Desarrollar transportes urbanos de calidad

- Poner la tecnología al servicio de transportes limpios y eficaces

- Gestionar los efectos de la mundialización

- Desarrollar los objetivos medioambientales a medio y largo plazo para un sistema de transporte sostenible

ESPAÑA

\section{EL TRANSPORTE DE MERCANCÍAS POR CARRETERA EN}

En España, en 2005, el transporte ocupó a 938.526 personas, de las que $730.521(77,8 \%)$ eran asalariados y generó un valor añadido de 34.178 millones de euros, lo que supone el 6,25\% del VAB del sector servicios y el 3,8\% del PIB nacional a precios de mercado (INE: Encuesta Anual de Servicios 2005; INE: Contabilidad Nacional de España, 2005). Según los últimos datos disponibles (Roza Braga 2007), la carretera supone el 80,3\% del consumo final del sector seguida del transporte aéreo $(12,5 \%)$, marítimo $(4,3 \%)$ y ferroviario $(2,9 \%)$. 
Como podemos observar en el Esquema 1, la Clasificación Nacional de Actividades Económicas (CNAE-93 Rev.1) recoge las diferentes clases de transporte, no en función de "lo transportado" (viajeros o mercancías), sino en función del modo utilizado (tubería, ferrocarril, avión, etc.). Así, dentro del Grupo I Transporte, almacenamiento y comunicaciones, el subgrupo 60 engloba el Transporte terrestre y transporte por tubería y la subclase 60.24 recoge, específicamente, el Transporte de mercancías por carretera.

Esquema 1: Actividades incluidas en el Grupo I Transporte, almacenamiento y comunicaciones

60 Transporte terrestre y transporte por tubería

60.1 Transporte por ferrocarril

60.2 Otros tipos de transporte terrestre

60.21 Otros tipos de transporte terrestre regular de viajeros

60.22 Transporte por taxi

60.23 Otros tipos de transporte terrestre discrecional de viajeros

60.24 Transporte de mercancías por carretera

60.3 Transporte por tubería

61 Transporte marítimo, de cabotaje y por vías de navegación interiores

62 Transporte aéreo y espacial

63 Actividades Anexas a los Transportes, Actividades de Agencias de Viajes.

64 Correos y Telecomunicaciones.

Fuente: CNAE.

A pesar del destacado peso que el transporte público y discrecional de viajeros tiene en España, las mercancías generan alrededor de las tres cuartas partes (75\% en 2005) del valor añadido que el transporte por carretera genera (Gráfico 2).

Como se ha comentado a escala europea, el crecimiento del peso de la carretera es también en España una tendencia sostenida desde principios de los años ochenta, debido tanto al aumento de las tn-km generadas (110.500 millones de tn-km en 1985, 367.497 millones de tn-km en 2005), como al estancamiento en otros modos (12.074 millones tn-km producidas por el ferrocarril en 1985, 11.641 millones tn-km en 2005) ${ }^{1}$. En el Gráfico 3, que muestra las distribución del transporte de mercancías en función del modo utilizado, apreciamos cómo en 2004 la carretera generó 
el $84 \%$ de las tn-km producidas en el conjunto del transporte interior interurbano español $\mathrm{y}$, sin embargo, en los movimientos internacionales el transporte marítimo es claramente preponderante, aunque la carretera ha ido aumentando su presencia a lo largo de los años, pues ha pasado de movilizar el 5,6\% de las toneladas transportadas en 1975 al 26,6\% en 2004.

Gráfico 2: Distribución del VABpm del transporte por carretera. España. 2000-05. Porcentajes

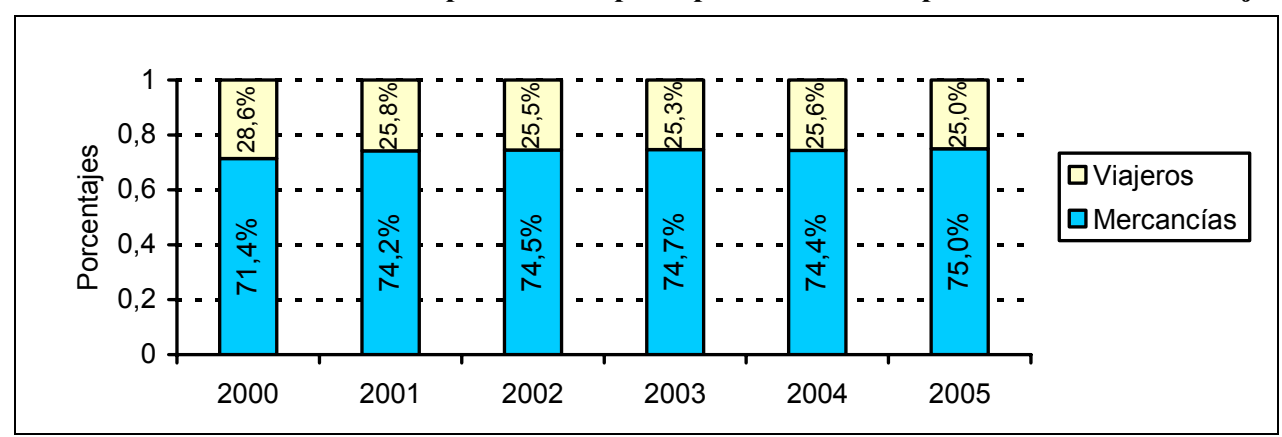

Fuente: Elaboración propia a partir de INE (varios años) Encuesta Anual de Servicios.

Gráfico 3: Distribución por modos del transporte de mercancías. España. 2004

Transporte interior interurbano Porcentaje sobre el total anual de tn-km

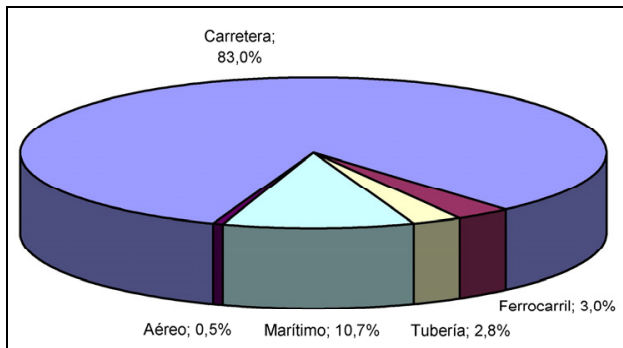

Transporte internacional Porcentaje sobre el total anual de tn

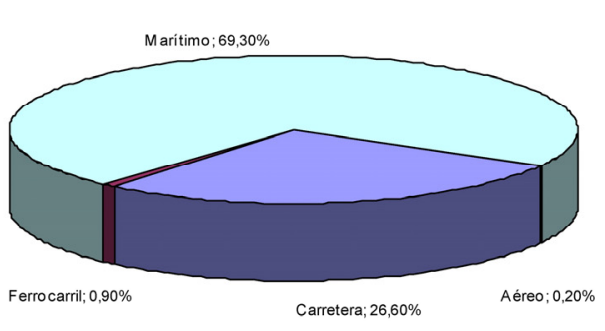

Fuente: Ministerio de Fomento: Observatorio del Transporte de Mercancias por Carretera 2007.

Cuadro 1: Número de empresas. 2005. España

\begin{tabular}{|l|r|c|}
\hline Ámbito & Número & Porcentaje \\
\hline Transporte de mercancías por carretera & 135.549 & 67,19 \\
\hline Transporte urbano e interurbano por autobús & 4.496 & \\
\hline Transporte por taxi & 61.680 & \\
\hline Transporte por carretera & 201.725 & 99,86 \\
\hline TOTAL TRANSPORTE (Viajeros + mercancías) & 202.004 & 100,0 \\
\hline
\end{tabular}

Fuente: INE: Encuesta Anual Servicios 2005. 
En cuanto a la estructura empresarial, el número de empresas dedicadas al transporte por carretera es muy alto en relación a la población que ocupan y al valor añadido y a la cifra de negocio que generan. Hasta tal punto que, según CONSULTRANS, España es el país con mayor número de empresas de transporte terrestre y de transporte por carretera de la UE. Como observamos en el Cuadro 1, el 99,86\% de las empresas dedicadas al transporte se dedica al transporte por carretera y el $67 \%$ al transporte de mercancías. Esta abundancia de empresas tan destacada en el transporte de mercancías se debe al elevado número de autónomos (principalmente conductores), que son la forma más frecuente de organización. A pesar de los cambios recientes en la regulación, otros modos competidores, como el ferrocarril, presentan una fuerte concentración industrial, lo cual genera importantes diferencias en la estructura de los mercados que se tornan fundamentales desde el punto de vista estratégico, por ejemplo, en la capacidad de las empresas de uno y otro modo para negociar precios.

El sector del transporte siempre ha sido muy intensivo en trabajo, y aún más el transporte terrestre, que es la rama de actividad con mayor número de ocupados. Como se desprende de los datos proporcionados por la Encuesta Anual de Servicios del INE, el mercado de trabajo del sector se caracteriza por escasa presencia del trabajo femenino (la participación de las mujeres en el empleo apenas alcanza el $10 \%$ del total) y un peso relativo de los asalariados (730.000 personas en 2005) bajo con respecto a la media nacional (77\% frente a $81,9 \%$, respectivamente) debido al elevado número de autopatronos. Por su parte, la tasa de paro en los transportes terrestres es también mucho más baja que la media nacional (4\% frente a $11 \%$ en 2004 ).

El futuro del sector en España está delimitado por el Plan Estratégico para el Sector del Transporte de Mercancías por Carretera (PETRA), cuyos pilares básicos son la seguridad, la calidad, la protección del entorno natural y una visión integral del sector. Los trabajos realizados durante el diseño del plan identificaron los siguientes campos donde son necesarias acciones prioritarias a corto plazo: reestructuración empresarial (dimensión), formación, nuevas tecnologías, imagen, logística y comercialización. En estos campos, el Plan prevé reforzar la debilidad estructural ampliando las ayudas para la reducción de autónomos, aumentar las ayudas para la formación de los conductores profesionales, difundir el uso de herramientas informáticas, crear observatorios, financiar estudios y apoyar la autorregulación, fomentar el uso del tacógrafo digital y mejorar el sistema de inspecciones, etc. Asimismo, se identificaron otros factores 
donde era necesario una actuación a medio plazo, a saber: ordenación del sector (modificación del marco jurídico), actuaciones socio-laborales, infraestructuras e intermodalidad, expansión exterior y mejoras medioambientales (renovación el parque, conducción ecológica...). Finalmente, el Plan PETRA propone un plan de seguimiento y control que permita a la Administración evaluar en cada momento la situación de cada uno de los proyectos y sus efectos en las áreas estratégicas de actuación.

\section{CASTILLA Y LEÓN}

\section{EL TRANSPORTE DE MERCANCÍAS POR CARRETERA EN}

Según la Contabilidad Regional Anual de Castilla y León, en 2005, la rama de actividad Transporte y Comunicaciones, produjo 3.402 millones de euros en la región (Gráfico 4), lo que supuso el 15,7\% del VAB generado por los servicios de mercado y el 7,46\% del VAB total regional a precios básicos. La rama ocupó a un total de 43.151 personas que suponían el $10,75 \%$ del empleo generado por los servicios de mercado y el $4,26 \%$ del empleo total ${ }^{2}$. Como podemos observar en el Gráfico 4, los Transportes y las Comunicaciones, sobre todo desde 1997, han crecido más que la economía en general, más que el sector Servicios de mercado y han sido cada vez responsables de una mayor generación de valor agregado (en 1997 aportaron el $6,89 \%$ del total y en 2002 el $7,85 \%$, en precios constantes y a precios básicos).

2 Ha de tener en cuenta el lector que la Contabilidad Anual de Castilla y León, al igual que la Contabilidad Regional de España, no proporciona cuentas desagregadas del sector Transporte y Comunicaciones, por lo que los datos anteriores incluyen los correspondientes a las ramas 63 (Actividades anexas a los transportes; actividades de agencias de viajes) y 64 (Correos y telecomunicaciones) de la CNAE-93. En 2000, según la Contabilidad Nacional, estas dos ramas supusieron el $56,31 \%$ del VAB y el $38,40 \%$ del empleo del total Transporte y Comunicaciones. Por otra parte, es necesario tener en cuenta que los datos proporcionados por la Junta de Castilla y León no coinciden con los del INE. Y dentro de este organismo, hay cifras de empleo muy dispares en función de que se utilice la serie 1995-2004 ó la 2000-2006. 
Gráfico 4: Evolución del VAB a precios básicos. Castilla y León 1995-2005. Euros corrientes 1995-2003. Euros constantes Números índice 1995=100

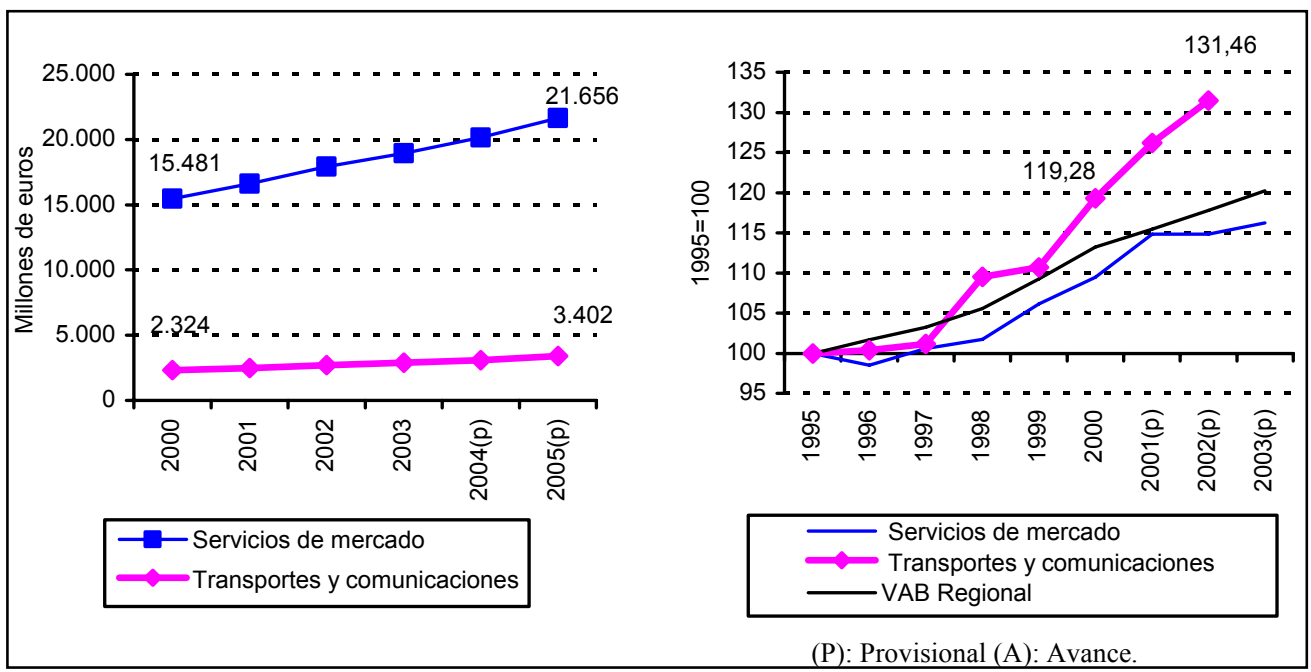

Fuente: Junta de Castilla y León (varios años) Contabilidad Regional Anual.

Fuente: INE (varios años) Contabilidad Regional de España.

Como venimos exponiendo y se puede observar en el Gráfico 5, la carretera es preponderante en el transporte de mercancías. En la Comunidad Autónoma de Castilla y León, en 2005, se transportaron por carretera un total de 141,46 millones de toneladas de mercancía $(100,55$ millones en 2000, 163,07 millones en 2006), frente a los 2,4 millones que se transportaron por ferrocarril ${ }^{3}$. Por otro lado, el ferrocarril muestra una tendencia muy estable, ligeramente descendente, mientras la carretera muestra un importantísimo avance, con crecimientos superiores al $8 \%$ anual en la última década, periodo en el que llaman la atención los años 1998 y 1999, cuando se incrementaron las mercancías transportadas por carretera en un $14,3 \%$ y un $18,5 \%$, respectivamente y los años 2002 y 2003 con crecimientos del $10 \%$ anual en ambos casos. Esta tendencia creciente se frena ligeramente en 2005, aunque visto el dato provisional de 2006 no parece que vaya a convertirse en un cambio sostenido. Por su parte, las

3 Dentro del Total transportado se incluyen las mercancías cargadas en la Comunidad Autónoma con destino al exterior de la región, las procedentes del exterior y descargadas en la Comunidad y el transporte intrarregional. En el caso del ferrocarril, no existen datos de transporte intrarregional de mercancías aunque, en ningún caso, la inclusión de estos flujos haría variar la importancia relativa de los distintos modos de transporte en la Comunidad. 
mercancías transportadas por avión son prácticamente insignificantes, pues apenas alcanzaron 306 toneladas en 2005.

Gráfico 5: Mercancías transportadas según modo de transporte. Castilla y León. 2000-2006. Millones de toneladas anuales

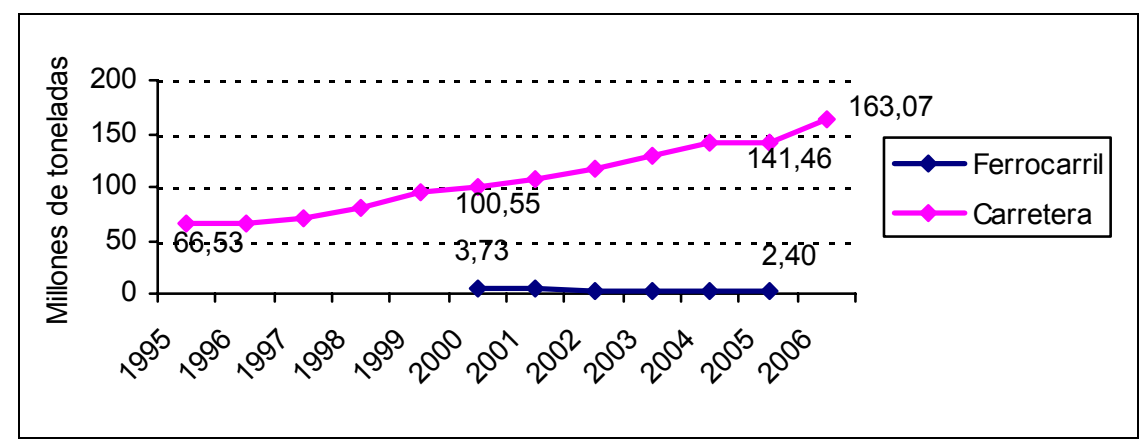

Fuente: Junta de Castilla y León (2006) Anuario Estadístico y Ministerio de Fomento (2006) Encuesta Permanente del Transporte de Mercancias por Carretera.

\section{Parque, matriculaciones y autorizaciones}

Con una tendencia inequívocamente creciente, el parque de camiones y tractores industriales de Castilla y León ha aumentado de 71.493 vehículos en 1997 a 149.692 en 2006 , lo que supone un incremento total del $109 \%$ a una tasa media del $9,7 \%$ anual; y ha crecido tanto el número de camiones no articulados como el de cabezas tractoras. Según los últimos datos, estos vehículos suponen el 9,67\% del parque móvil total de la Comunidad, y el 5,6\% del parque móvil nacional de vehículos pesados (Gráfico 6).

Gráfico 6: Parque de camiones y tractores industriales de Castilla y León Unidades de vehículos. 1997-2006 Distribución provincial. Porcentajes. 2006

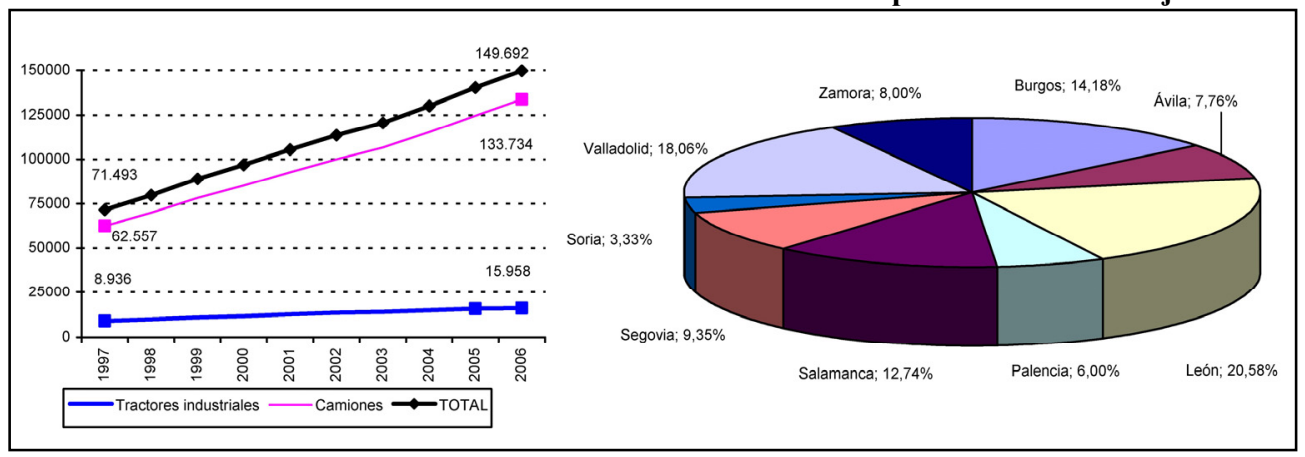

Fuente: DGT: Estadísticas definitivas. 
Las matriculaciones (Gráfico 7) de camiones en Castilla y León se han mantenido muy estables en los últimos años, en torno a los 1.270 vehículos anuales, con una ligera tendencia decreciente entre 1999 y 2002 que parecía estar recuperándose. La distribución provincial del parque y las nuevas matriculaciones es muy similar y destaca cómo, aún sin ser la provincia más poblada ni la de mayor actividad, León aglutina más del $20 \%$ del parque de camiones y tractores industriales y el $18 \%$ de las matriculaciones, seguida de Valladolid y Burgos. En cualquier caso, merece la pena llamar la atención sobre los datos de Salamanca, Palencia y Ávila, que muestran mayor peso en la distribución de las matriculaciones que en la del parque.

Gráfico 7: Matriculaciones de camiones en Castilla y León

Unidades de vehículos. 1998-2006

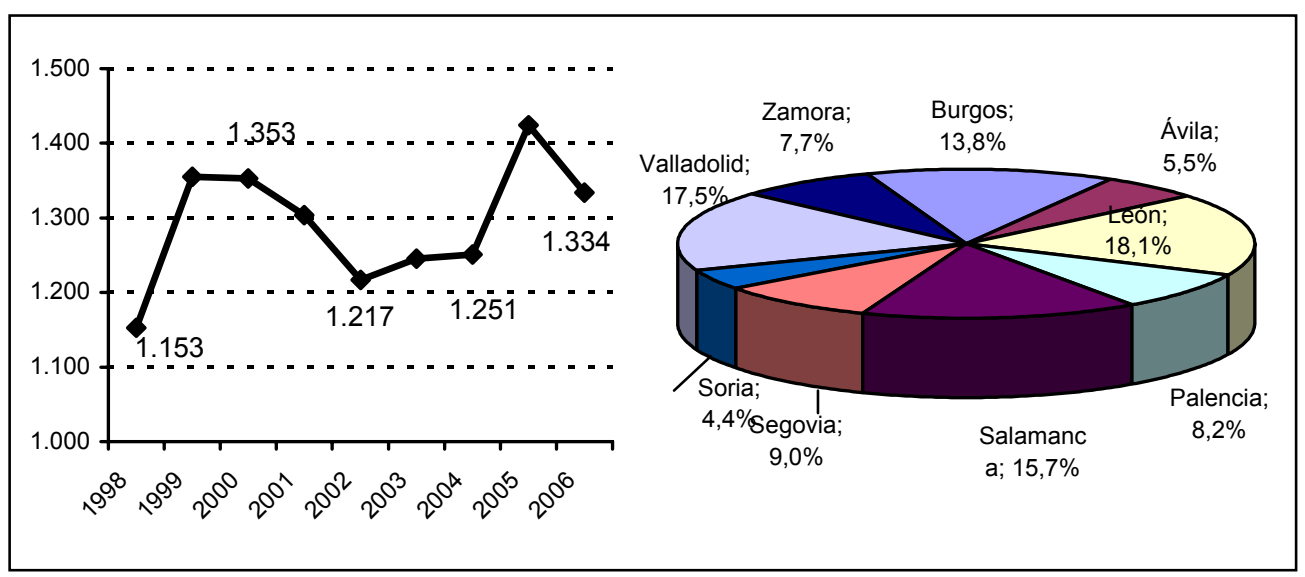

Fuente: DGT: Estadísticas definitivas.

La distribución de los vehículos en función del combustible utilizado muestra importantes disparidades con respecto al conjunto de España, como se puede observar en el Gráfico 8. Mientras que en España el número relativo de camiones que utilizan gas-oil se ha situado por encima del $90 \%$ del total desde el primer año de referencia (1997), en Castilla y León, aún en 2005 , este porcentaje apenas alcazaba el $85 \%$. No obstante, gracias al constante aumento de los vehículos de de gas-oil, cercano al $5,6 \%$ anual y a la incesante disminución de aquellos que utilizan gasolina, las diferencias han ido disminuyendo paulatinamente desde los 17 puntos porcentuales de finales de los años noventa a los 11 puntos porcentuales de 2005. En el caso de los tractores industriales, no se aprecia tanta 
divergencia y tanto en Castilla y León como en el conjunto de España, prácticamente el $100 \%$ de ellos utiliza gas-oil.

Gráfico 8: Distribución de los vehículos pesados en función del combustible utilizado. 2006. Porcentajes

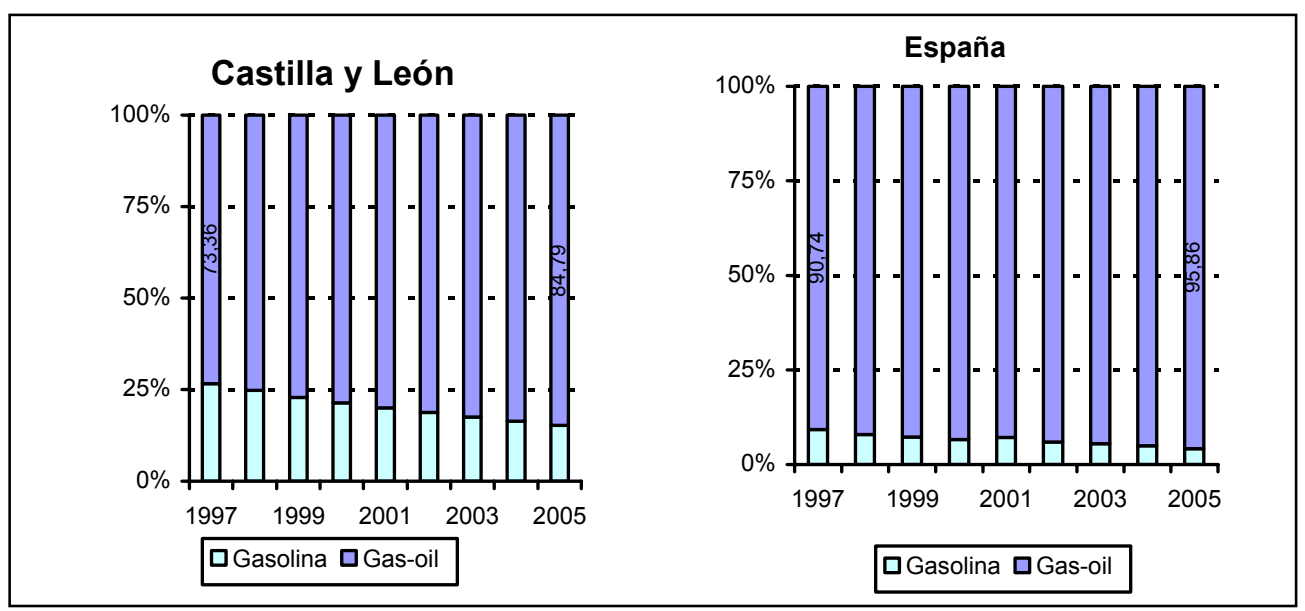

Fuente: DGT: Estadísticas definitivas y Anuario Estadístico General 2005.

\section{Estructura empresarial}

Según el Ministerio de Fomento, en diciembre de 2007, en Castilla y León estaban instaladas 6.540 empresas de transporte pesado público y 7.658 de transporte privado de mercancías (el $8,6 \%$ y el $7,8 \%$, respectivamente, del total nacional). La distribución provincial puede observarse en el cuadro y gráficos adjuntos. A 1 de enero de 2007, el número total de vehículos pesados con autorización para el transporte de mercancías por carretera en servicio público en Castilla y León ascendía a 18.415 , un $5,7 \%$ más que el año anterior. Estas autorizaciones, que suponen el 7,2\% del total nacional, son en el $89,3 \%$ de los casos de ámbito nacional y en el $10,7 \%$ de ellos de ámbito comarcal o local. Esta distribución, al igual que en años anteriores, es algo diferente a la media nacional, donde del total de autorizaciones, sólo el $80 \%$ son de ámbito nacional y el $20,7 \%$ son ámbito de ámbito comarcal o local ${ }^{4}$.

4 Por efecto de lo dispuesto en el Real Decreto 1225/2006 de 27 de octubre, no se otorgan nuevas autorizaciones de transporte público de mercancías en vehículos pesados de ámbito local a partir del 16 de noviembre de 2006. 
Cuadro 2 y Gráfico 9: Empresas de Transporte de Mercancías. Castilla y León y provincias, 2007 Servicio Público Pesado y

Servicio Público Pesado. Servicio Privado Distribución provincial (\%)

\begin{tabular}{|l|r|r|}
\hline & $\begin{array}{c}\text { Serv. público } \\
\text { PESADO }\end{array}$ & $\begin{array}{c}\text { Serv. privado } \\
\text { Mercancías }\end{array}$ \\
\hline Ávila & 407 & 673 \\
\hline Burgos & 1.307 & 997 \\
\hline León & 1.354 & 1.390 \\
\hline Palencia & 651 & 613 \\
\hline Salamanca & 538 & 1.185 \\
\hline Segovia & 532 & 663 \\
\hline Soria & 291 & 349 \\
\hline Valladolid & 965 & 1.081 \\
\hline Zamora & 495 & 707 \\
\hline CyL & 6.540 & 7.658 \\
\hline ESPAÑA & 76.002 & 96.071 \\
\hline
\end{tabular}

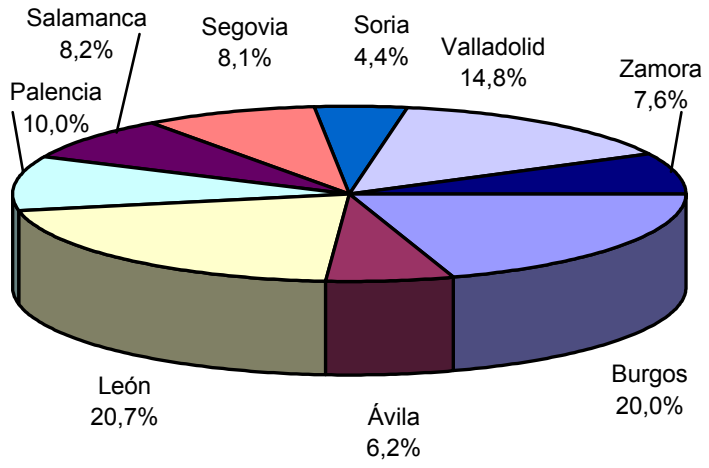

Fuente: Ministerio de Fomento (2007).

Cuadro 3: Autorizaciones a empresas de Transporte Pesado de Mercancías. Servicio Público. Castilla y León, provincias. 2006

\begin{tabular}{|l||r|r|r||c|}
\hline & Nacional & Comarcal & Local & $\begin{array}{c}\text { Media autorizaciones } \\
\text { nacionales por empresa }\end{array}$ \\
\hline Ávila & 959 & 53 & 32 & 2,36 \\
\hline Burgos & 3.570 & 78 & 91 & 2,73 \\
\hline León & 3.365 & 174 & 148 & 2,49 \\
\hline Palencia & 1.587 & 50 & 46 & 2,44 \\
\hline Salamanca & 1.882 & 79 & 69 & 3,50 \\
\hline Segovia & 1.535 & 35 & 52 & 2,89 \\
\hline Soria & 940 & 26 & 8 & 3,23 \\
\hline Valladolid & 3.571 & 131 & 71 & 3,70 \\
\hline Zamora & 1.321 & 63 & 55 & 2,67 \\
\hline Castilla y León & 18.730 & 689 & 572 & 2,86 \\
\hline ESPAÑA & 253.599 & 9.906 & 17.538 & 3,34 \\
\hline
\end{tabular}

Nota: Dada la imposibilidad de discriminar el servicio privado ligero del pesado, mostramos únicamente las autorizaciones del servicio público de vehículos pesados. Fuente: Ministerio de Fomento (2006).

Con respecto a este tema y aunque no hay datos a escala regional, es necesario hacer notar que la estructura del sector, aún teniendo en cuenta los cambios legislativos recientes, está experimentando una notable concentración empresarial, pues el número de empresas en España con más de cinco autorizaciones ha crecido un $94,7 \%$ entre 1999 y 2007, mientras que el número de empresas con menos de cinco autorizaciones ha decrecido un 10\% (Roza Braga 2007). Como se puede observar en el 
Cuadro 3, la media de autorizaciones nacionales por empresa se sitúa entre dos y tres, por debajo de la media nacional. Las provincias que disponen de mayor número de autorizaciones son León, Valladolid y Burgos, pero las mayores empresas parecen estar en Valladolid y Salamanca, con una media de 3,7 y 3,5 autorizaciones por empresa.

\section{Cantidad, origen y destino de los flujos}

Como vemos en el Gráfico 10, la mercancía transportada ha crecido considerablemente desde mediados de la década pasada, y si sólo nos fijamos en los últimos cinco años, los flujos intrarregionales lo han hecho a una tasa media anual del 7,9\% y los interregionales a un $7 \%$ en el caso de las mercancías expedidas y a un $4,9 \%$ en el caso de las recibidas. Esto significa tasas de crecimiento anuales que, a pesar de ser negativas en dos casos puntuales (1996 y 2001 en flujos interregionales), en 9 ocasiones superan el $10 \%$ y en casi todas son mayores que el crecimiento nominal de la economía.

Gráfico 10: Transporte de mercancías por carretera. Flujos intrarregionales e interregionales. Castilla y León. 1995-2006. Millones de toneladas

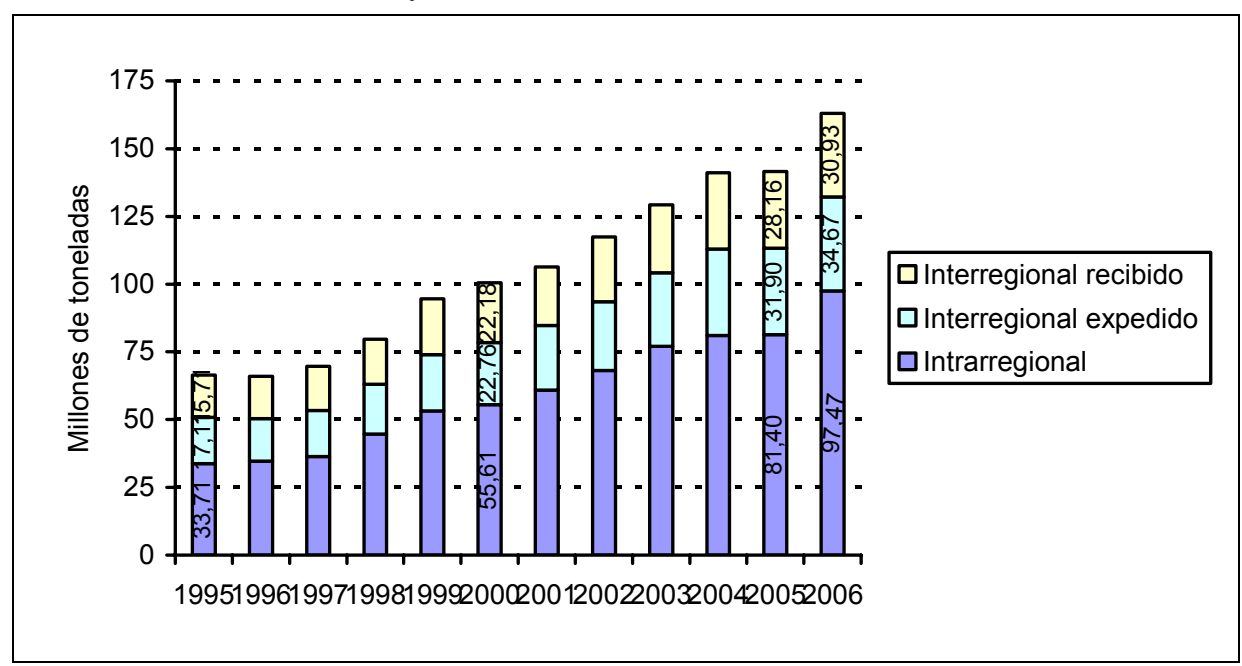

Nota: Los flujos intrarregionales no incluyen aquellos intramunicipales.

Fuente: Ministerio de Fomento (varios años) Encuesta Permanente del Transporte de Mercancías por Carretera, y Junta de Castilla y León: Anuario estadístico.

Como resultado de esta evolución, en 2006 se realizaron 19,5 millones de operaciones de transporte de mercancía con origen $\mathrm{y} / \mathrm{o}$ destino 
en Castilla y León, en las que se transportaron 165 millones de toneladas, alrededor del 8,3\% del total nacional. El 59\% de las toneladas transportadas (Gráfico 11) se debe a flujos intrarregionales, el 21\% a flujos con origen en la Comunidad Autónoma y el $19 \%$ a flujos cuyo destino final está en su territorio. El peso relativo de las operaciones internacionales es mucho menor en Castilla y León que en España (1,7\% y 2,8\%, respectivamente), hecho que muestra una menor relación directa con mercados extranjeros que otras Comunidades Autónomas.

Gráfico 11: Distribución de las mercancías transportadas en función de su origen y destino. Castilla y León. 2006. Porcentajes sobre el total de toneladas transportadas

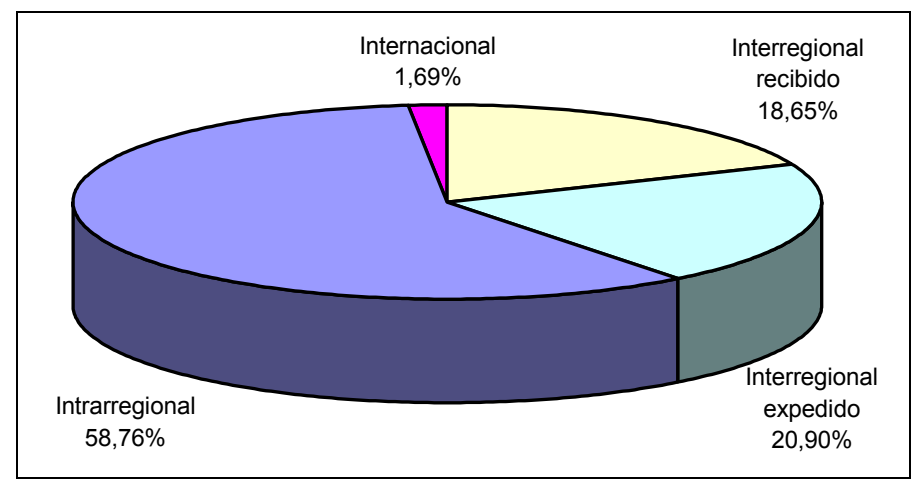

Fuente: Ministerio de Fomento (2006) Encuesta Permanente del Transporte de Mercancias por Carretera.

En Castilla y León, las toneladas de mercancía expedidas superan en un $12 \%$ a las recibidas, lo cual muestra una mayor capacidad de producción que de consumo. Otras Comunidades Autónomas presentan resultados muy diferentes; por ejemplo Madrid, recibe un $50 \%$ más de lo que expide, o Castilla-La Mancha, donde se expiden un 30\% más de mercancías de las que se reciben ${ }^{5}$.

En 2006, siguiendo la tónica de los últimos años, un $26 \%$ de las mercancías cargadas en Castilla y León con destino nacional se dirigieron a Madrid, un $10 \%$ al País Vasco y sendos $8 \%$ a Cantabria, Castilla-La Mancha

5 Esta situación parecería fácil de explicar por la diferencias en el tamaño de los mercados, sin embargo aparecen otras Comunidades Autónomas, como por ejemplo Extremadura, con mercados relativamente pequeños y dónde la mercancía expedida es inferior a la recibida en un $30 \%$, lo cual podría ser un primer indicador de la escasa capacidad de producción y distribución. 
y Galicia. También son mencionables las relaciones (alrededor de 6\% del total en cada caso) con Asturias, Cataluña y la Comunidad Valenciana.

Cuadro 4: Operaciones de transporte de mercancías por carretera según tipo de desplazamiento. Castilla y León y España. 2006.

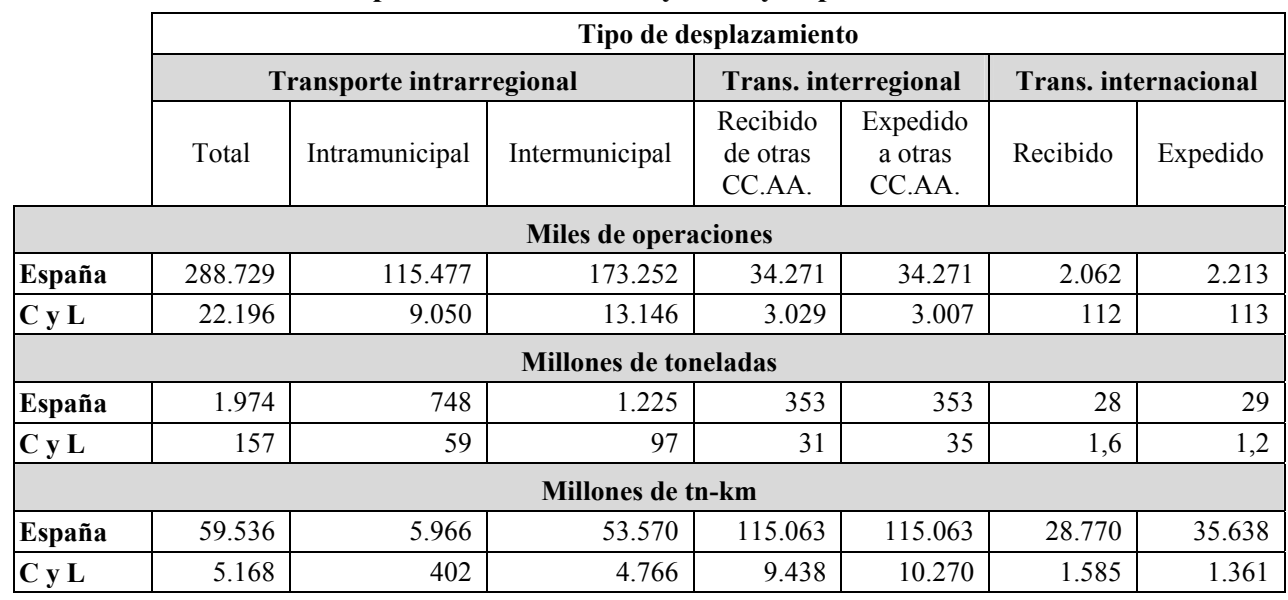

Fuente: Ministerio de Fomento: Encuesta permanente del transporte de mercancías por carretera.

En sentido contrario, la Comunidad de Madrid y el País Vasco son los principales proveedores de mercancías de Castilla y León, pues de allí parten el $16 \%$ de las mercancías recibidas, respectivamente. Les siguen Asturias, Galicia, Castilla-La Mancha y Cantabria, de donde parten el $11 \%$, el $9 \%$ y sendos $7 \%$, respectivamente.

Gráfico 12: 2006. Porcentajes sobre las toneladas expedidas a/ recibidas de todas Comunidades Autónomas desde Castilla y León

Principales Comunidades Autónomas DESTINO de las mercancías

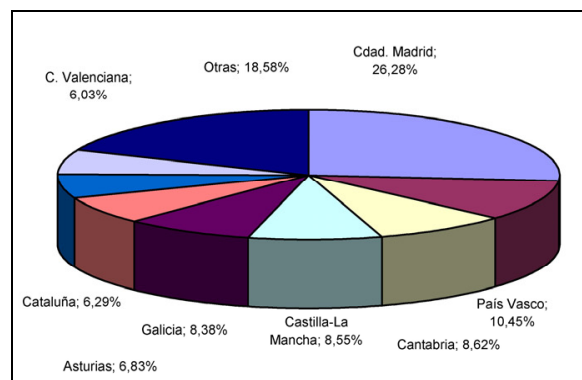

Principales Comunidades Autónomas ORIGEN de las mercancías

Fuente: Elaboración propia a partir de Ministerio de Fomento: Encuesta permanente del transporte de mercancias por carretera. 
De esta forma, y como parece lógico por los volúmenes de población y actividad, la Comunidad de Madrid se revela, en términos relativos, muchísimo mejor consumidor que proveedor, al contrario de lo que ocurre con Asturias y el País Vasco.

En los mapas que ofrecemos a continuación se puede ver, en 2006, esta relación entre Castilla y León y el resto de comunidades autónomas.

De todas las operaciones realizadas, tanto a escala nacional como autonómica, casi la mitad (47\%) se efectúan en vacío, es decir, son desplazamientos en los que no se transporta ningún tipo de carga. Como muestra el Gráfico 13, este tipo de operaciones, que constituye una de las mayores preocupaciones de las empresas de transporte, se hace más habitual a medida que se reduce la longitud del desplazamiento, pues alcanza el $48,9 \%$ de las operaciones intrarregionales, el $33 \%$ de las interregionales y como parece lógico, se reduce a menos del $20 \%$ de las internacionales. No obstante, la Encuesta Permanente del Ministerio de Fomento reconoce que no todas estas operaciones pueden ser interpretad[as] como retornos "completos" en vacío, pues los vehículos realizan pequeños recorridos de operaciones en vacío para cargar y evitar de ese modo volver al punto de origen sin carga (Ministerio de Fomento 2005b).

Gráfico 13: Operaciones en vacío en el transporte de mercancías por carretera por tipo de desplazamiento. España y Castilla y León. 2006. Porcentajes sobre las operaciones totales por tipo de desplazamiento

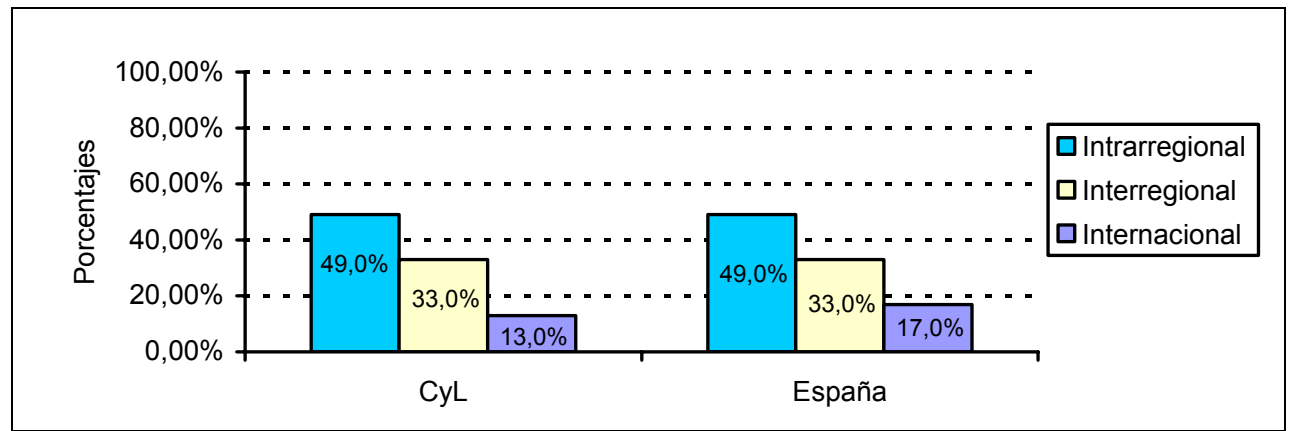

Fuente: Elaboración propia a partir de Ministerio de Fomento (2007) Encuesta permanente del transporte de mercancías por carretera. 
Mapa 1: Flujos interregionales de mercancías. Castilla y León. 2006.
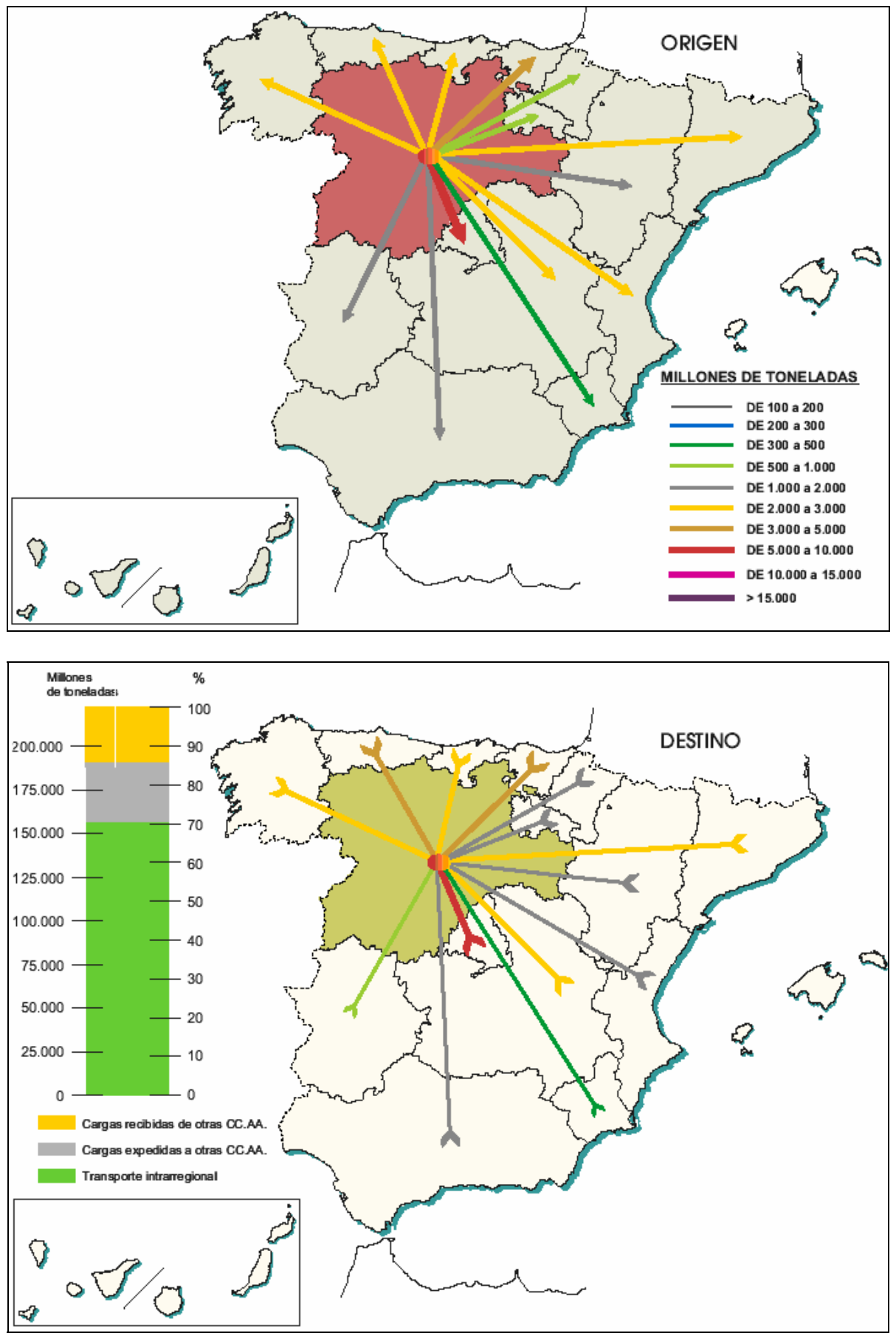

Fuente: Ministerio de Fomento (2007) Encuesta permanente del transporte de mercancías por carretera. 


\section{Distribución por tipo de mercancía}

Como muestra el Gráfico 14, en 2006, del total de operaciones realizadas con carga, en el $51 \%$ de las ocasiones se transportaban "Minerales y materiales para la construcción", en el $17 \%$ "Máquinas, vehículos, objetos manufacturados" y en el $11,5 \%$ de las ocasiones, con una tendencia decreciente, "Productos alimenticios y forrajes". Este patrón se reproduce en casi todas las divisiones de los desplazamientos (especialmente en el transporte intramunicipal), si bien en las operaciones intrarregionales también cobran cierta importancia (13,5\% del total de operaciones) los "Productos agrícolas y animales vivos" en detrimento de "Máquinas, vehículos, objetos manufacturados". Una situación muy similar se refleja si analizamos las toneladas transportadas, pero si hacemos el análisis teniendo en cuenta las tn-km, las "Máquinas, vehículos y objetos manufacturados" y los "Productos alimenticios y forrajes" adquieren mucha más importancia $(23,5 \%$ y $21,2 \%$, respectivamente) en detrimento de los "Minerales y materiales para la construcción" que sólo representan el $24,7 \%$ de las $\mathrm{tn}-\mathrm{km}$ producidas aunque mantienen su preponderancia en el transporte intramunicipal ${ }^{6}$.

Gráfico 14: Distribución por clase de producto del transporte de mercancías. Castilla y León. 2006. Porcentajes del total de operaciones con carga y de las tn-km

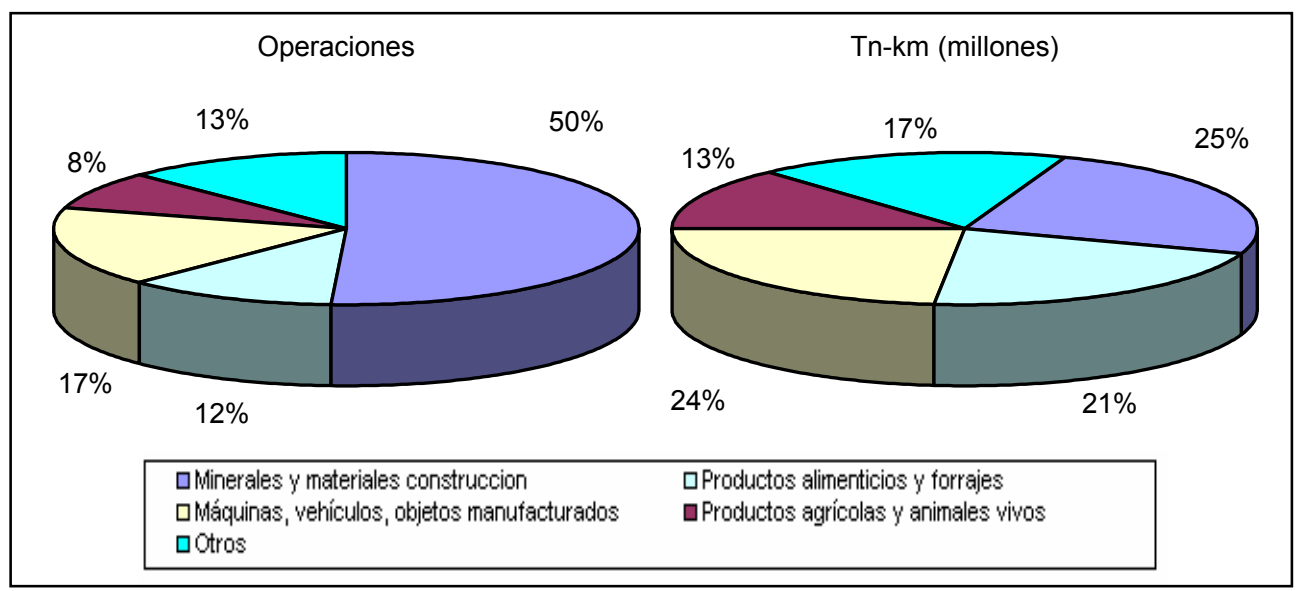

Fuente: Ministerio de Fomento (2007) Encuesta permanente del transporte de mercancias por carretera. 


\section{CONCLUSIONES}

La rama Transportes y Comunicaciones generó, en 2005, 3.402 millones de euros, que suponen el 7,46\% del VAB total de Castilla y León a precios básicos y ocupó a 43.151 personas, que suponen el $4,26 \%$ del empleo total de la Comunidad Autónoma. Al igual que en otros ámbitos geográficos, el sector ha crecido más que la economía en general, más que el sector Servicios de mercado y ha sido cada vez responsable de una mayor generación de valor agregado.

La carretera es el modo preponderante en el transporte de mercancía, con crecimientos superiores al $8 \%$ anual en la última década. En 2007, estaban instaladas en Castilla y León alrededor de 6.540 empresas de transporte pesado público que poseían alrededor de 20.000 autorizaciones, casi todas nacionales. El parque móvil de camiones y tractores industriales ascendía en 2006 a 149.000 vehículos, que representan el 5,6\% del parque móvil nacional de vehículos pesados.

En 2006 se realizaron 19,5 millones de operaciones de transporte de mercancía con origen y/o destino en Castilla y León, en las que se transportaron 165 millones de toneladas, alrededor del 8,3\% del total nacional. Los flujos intrarregionales generan la mayor parte de las toneladas transportadas (59\%) y, entre los interregionales, son mayores las toneladas de mercancía expedidas que las recibidas así como sus correspondientes tasas de crecimiento.

Aunque en magnitudes diferentes, Madrid y el País Vasco son tanto los principales destinos de la mercancía expedida como los principales orígenes de la recibida.

En el $50 \%$ de las operaciones realizadas con carga se transporta "Minerales y materiales para la construcción", aunque en Tn$\mathrm{km}$ producidas ganan mucho peso las "Máquinas, vehículos, objetos manufacturados" y los "Productos alimenticios y forrajes".

\section{BIBLIOGRAFÍA}

COMISIÓN DE LAS COMUNIDADES EUROPEAS (2001) Libro Blanco la política Europea de transportes de cara al 2010: la hora de la verdad. 
CONSULTRANS (¿2005?) Estudio Socio-Económico del Transporte por Carretera en España.

DIRECCIÓN GENERAL DE TRÁFICO: Estadísticas definitivas. www.dgt.es.

- (¿2006?) Anuario Estadístico General 2005. www.dgt.es.

EUROPEAN FOUNDATION FOR THE IMPROVEMENT OF LIVING AND WORKING CONDITIONS (2004) Sector Futures. Transport: Where are we going? www.eurofound.eu.int.

INE: Contabilidad Nacional de España. www.ine.es

-: Contabilidad Regional de España. www.ine.es

- (varios años) Encuesta Anual de Servicios. www.ine.es

JUNTA DE CASTILLA Y LEÓn (varios años) Anuario Estadístico.

- (varios años) Contabilidad Regional Anual.

MINISTERIO DE FOMENTO (2001) Plan estratégico para el sector del transporte de mercancías por carretera (PETRA). Dirección General de Transportes por Carretera.

- (varios años) Encuesta Permanente del Transporte de Mercancías por carretera.

- (2005) Evolución de los Indicadores Económicos y Sociales del Transporte por Carretera. Secretaría General de Transportes. Dirección General de Transportes por Carretera.

-: Observatorio del Transporte de Mercancías por Carretera, varios Años. Secretaría General de Transportes. Dirección General de Transportes por Carretera.

- (2007) Información estadística. Datos del registro general. www.fomento.es.

RoZA BRAGA, E. (2007) Evolución del transporte de mercancías por carretera desde la liberación (años 1999-2007). www.cetcat.com/cetcat/. 